\title{
Chemokine Receptor
}

National Cancer Institute

\section{Source}

National Cancer Institute. Chemokine Receptor. NCI Thesaurus. Code C18018.

Encoded by Chemokine Receptor Genes (GPCR Family), cell surface Chemokine

Receptors are seven-transmembrane $\mathrm{G}$ protein-coupled receptor glycoproteins that bind to chemokines and mediate the migration of pro-inflammatory cells. Like chemokines, the chemokine receptors can be divided, according to variations in a shared cysteine motif, into at least three structural branches: CR, CCR, and CXCR. (NCl) 